\title{
Stylistic Analysis of Philippine English and Singaporean English in Automotive Review Articles
}

Eduardo Teodoro B. Ramos, Jr.

MFI Polytechnic Institute, Inc., Philippine

edteo.ramos@yahoo.com

Al Ryanne G. Gatcho

Philippine Normal University, Philippine

gatcho.arg@pnu.edu.ph

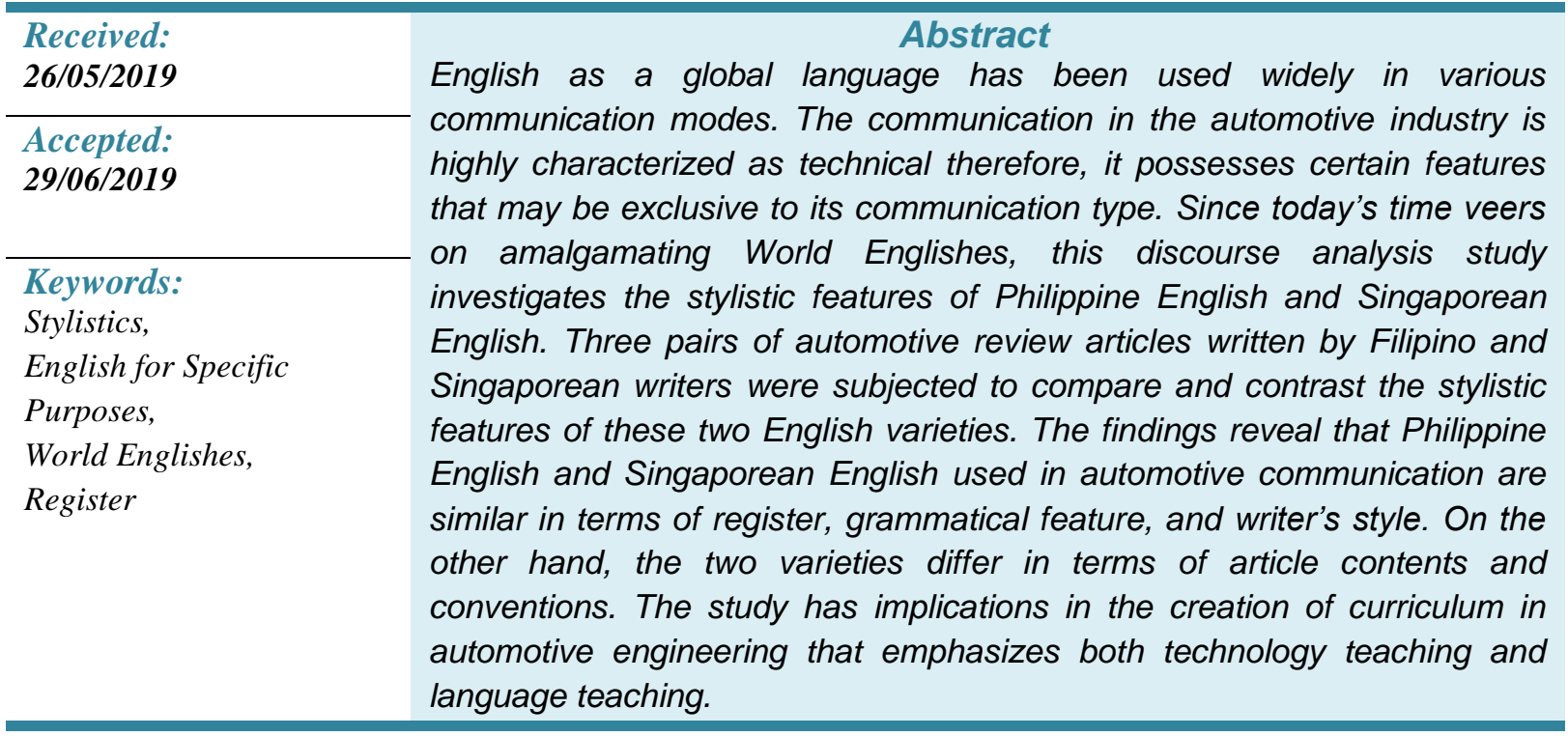

\section{INTRODUCTION}

One of the major automotive markets in the world is Southeast Asia, where industries are supported and regulated by the Association of Southeast Asian Nations (ASEAN). In recent decades, the ASEAN has been improving in terms of its economy, especially in the automotive industry context, because more consumers are able to purchase vehicles, apart from other accoutrements and necessities such as real properties, insurance policies, and even modern gadgets. Various multinational automotive companies have established international factories to implement cost-saving measures and to maximize profits without compromising on vehicle safety and comfort (Oxford Business Group, 2015; Remo, 2015).

Automotive reviews are normally written in English because many automotive-related terminologies- especially those pertaining to technology- are in English and do not have direct translations. Moreover, automotive reviews are often written in English, depending on the variety of English used by a writer. They are generally considered feature articles because they fascinatingly talk about their interesting facets instead of reporting about their world debut or 
Stylistic Analysis of Philippine English and Singaporean English in Automotive Review Articles

other pertinent news. Writers of automotive reviews are motoring journalists who are well-versed in the technical aspects of every vehicle they test drive and feature in their articles. Every automotive review is generally composed of the following components: a) introduction, b) technical specifications, c) body, and d) conclusion. The introduction discusses a brief history of the vehicle as well as the writers' experiences with the vehicle. Technical specifications contain figures and other information pertaining to the vehicle being reviewed; they are usually located on the right side of the article. The body of an automotive review contains descriptions and reviews on a vehicle's interior, exterior, driving performance (includes road feel, handling, and other factors) based on the writer's experiences. Finally, the conclusion of an automotive review summarizes the writer's assessment of a vehicle and includes recommendations for potential buyers and the respective manufacturers.

Rajagopalan (2004) asserts that World Englishes enables every communicator to establish his/her identity and become at par with the rest of the world at the same time in terms of communicative competence, which also applies to writing ${ }^{[7]}$. In the ASEAN context, Southeast Asian Englishes belong to what is considered the outer circle, which includes countries that use English as a second language and an official language of government, education, commerce, and technology (Deocampo, 2012; Salazar, 2014; Crystal, 2003; Rajagopalam, 2004); Philippine English and Singapore English are influenced by American English and by British English, respectively, in terms of lexicon, accent, and writing style (Salazar, 2014 and Pakir, 2009). Focusing on language as part of one's culture, Tan (2014) considers function and expertise (p. 23) as main factors in the success of one's transmission of his/her message to his/her interlocutors, whether in speech or writing, within one's own culture and across all other cultures. This applies most especially to the automotive field, which is considered highly technical and at the same time utilitarian since it serves one of the basic, modern societal needs: transportation. In terms of commonality of vehicle models, Singapore and the Philippines have many similarities, though some of these models vary slightly in terms of nomenclature and model trims (also known as variants). However, there are some vehicle models sold in Singapore that are not sold in the Philippines due to variances in the target market and in the salability of these vehicles, as well as the availability of spare parts and accessories. Other reasons include variances in tariff rates and conduciveness in organizing and sustaining regional headquarters and parts depots. A notable difference between the Philippines and Singapore is the presence of automotive facilities, which Singapore does not have due to its diminutive land area. Despite Singapore's size, it serves as a strategic location for regional headquarters and parts depots of non-Asian automotive brands (Barbour-Lacey, 2015 and Singapore Economic Development Board, 2015). Contrastively, the Philippines is considered a strategic location of several manufacturing facilities operated by some automotive companies, such as Isuzu, Mitsubishi, Toyota, and Nissan.

Therefore, to explicate on the intricacies of Philippine English and Singaporean English, this study unravels the stylistic features of automotive review articles in order to see the discrepancies and commonalities of these two varieties of English. The current study is anchored on Halliday's (in Hyland, 2005) systemic functional linguistics and stylistics theory. It seeks to answer the following research questions:

1. What stylistic similarities do Singaporean English-language and Philippine English-language automotive reviews have? 
2. What stylistic differences do Singaporean English-language and Philippine English-language automotive reviews have?

\section{METHODOLOGY}

The researchers collected three (3) pairs of automotive review articles written in English by Singaporean English (SE) and Philippine English (PE) writers. The articles, dating from 2017 to 2018 were sourced from online magazines and from online versions of leading automotive magazines both in the Philippines and Singapore. All articles were written by separate authors. In terms of the featured models, vehicle marques and models common to both the Philippines and Singapore, such as Toyota Vios, Chevrolet Orlando, and Volkswagen Golf, were chosen for each pair of articles pertaining to a vehicle to be consistent in terms of content related to vehicle features, performance, and other similar information. All articles were assigned alphanumeric codes; for example, SG-01 was assigned to the SE version of the first pair of automotive reviews; PH-01, for the first PE version. Discourse analysis, along with simple frequency, was utilized as a method of analysis for the current study since all articles were analyzed in terms of content. The data were limited to the following stylistic areas: register, grammar (adjective and pronoun usage), writers' style and points of view, and adherence to English variety conventions.

\section{RESULTS AND DISCUSSION}

\subsection{Similarities of Philippine English and Singaporean English \\ Register \\ Table 1. Registers Common across all Sample Automotive Reviews}

\begin{tabular}{|c|c|}
\hline $\begin{array}{c}\text { ACTUALREGISTERS } \\
\text { FOUND IN BOTH SE AND PE } \\
\text { AUTOMOTIVE REVIEWS }\end{array}$ & OPERATIONAL DEFINITION \\
\hline 1. Drive & $\begin{array}{l}\text { The layout related to the position of the wheels that control } \\
\text { the motion of a vehicle based on its connection to the engine. }\end{array}$ \\
\hline 2. Interior & The inside of a vehicle for drivers and passengers. \\
\hline 3. Steering & $\begin{array}{l}\text { The method of maneuvering a vehicle as well as the } \\
\text { component that determines the ease of turning a vehicle. }\end{array}$ \\
\hline 4. Injection & $\begin{array}{l}\text { The manner in which fuel is burned, in this case through } \\
\text { injectors found in a vehicle engine. }\end{array}$ \\
\hline 5. Turning radius & $\begin{array}{l}\text { Measures the degree of turning done by a driver to a vehicle, } \\
\text { especially when doing a u-turn or driving in a circular motion. }\end{array}$ \\
\hline 6. Wheelbase & The length of a vehicle from the front to the rear of a vehicle. \\
\hline 7. Displacement & The size of a vehicle engine, usually in cubic centimeters. \\
\hline 8. Grille & $\begin{array}{l}\text { A vehicle accessory found between headlamps that protects } \\
\text { the radiator and contributes to the aesthetics of a vehicle. }\end{array}$ \\
\hline 9. Headlamps & Front lamps that provide lighting especially at night. \\
\hline 10. MPV & $\begin{array}{l}\text { Acronym for multi-purpose vehicle, which is similar to a } \\
\text { wagon. }\end{array}$ \\
\hline 11. Sedan & A vehicle body type that has an enclosed, lengthened trunk \\
\hline
\end{tabular}


Stylistic Analysis of Philippine English and Singaporean English in Automotive Review Articles

\begin{tabular}{ll}
\hline 12. Suspension & (boot in British English). \\
\hline 13. Hatch & $\begin{array}{l}\text { A vehicle system located under the chassis that aids in vehicle } \\
\text { traction on pavements. }\end{array}$ \\
& $\begin{array}{l}\text { A contraction of the term hatchback, which is a vehicle that } \\
\text { has a door attached to the roof until the half of the vehicle tail. } \\
\text { It is different from a wagon because the rear cargo space is } \\
\text { limited. }\end{array}$
\end{tabular}

Based on the three pairs of online automotive reviews, the results on Table 1 revealed that the majority of the terms are exclusive to the automotive industry register. Contrastively, four of the terms found across all sample articles are words that are common in lay terms but have different definitions exclusive to the automotive industry (except for interior, which refers to the inside of an object). One of these examples is drive, which is usually defined as the controlling of a vehicle. The following instances of drive as a verb were observed:

Open the door and you are welcomed by Clark Plaid fabric with Alcantara bolstered seats for a nostalgic yet assuring feel when you drive it like you stole it. The red accents on the contrast stitching and ambient door trim accent lighting further remind you of the car's exciting nature. Sit on the driver's seat and hold the meaty flat bottom steering wheel and you'd find yourself wanting to awaken the monster inside. It comes with paddle shifters if you prefer to take shifting duties to your own.

(PH-01, Paragraph 4)

I have an embarrassing story to tell. Up till my first foray into the motoring journalism community, the Golf GTI was just another car. Sure, I've heard others rave about it for years since the Mk V version was launched but having not driven one personally up till then, I hadn't known what I was missing anyway.

Drive was found in the following sample paragraph as a noun:

(SG-01, Paragraph 1)

During long drives on the expressway, the Chevy can reach the usual cruising speeds of around 100kph. But past the three-digit figure, the engine will protest, the objection having nothing to do with you breaking the expressway's speed limit.

(PH-03, Paragraph 9)

As an adjective, the word drive was found below as a modifier of the word selector along with the word mode, being a type of selector that pertains to the available driving modes of modern vehicles equipped with automatic transmission:

The car comes with a drive mode selector allowing you to choose between Normal, Eco and Sport modes. The driving modes tweak engine maps, transmission shift points and throttle response, since it's a GTI, I kept it on "Sport" mode most of the time. Sport suspension comes standard as well as uprated (sic) brakes with red colored calipers to match the engine and complement the tires.

(PH-01, Paragraph 7)

In the case of drive as a technical term, the said word was found in the table of specifications of all sample articles. It pertained to the type of vehicle layout and to the positioning of the wheels that were responsible for vehicle traction, which was front-wheel drive in the case of all three vehicles that were the respective subjects of the articles. 
A second example of a term found across all sample articles was the word steering. The following excerpts used the term as a noun and as a reference to the system that is responsible for the handling and maneuvering of a vehicle:

Expectedly, the steering is light, which is ideal for negotiating the city traffic and parking, but feedback is lacking. Grip from the comfort-oriented Yokohama dB Decibel tyres is just adequate, but it makes up for it by working together with the suspension to equip the Vios with a relatively quiet and comfortable ride.

(SG-02, Paragraph 12)

The GTI interior remains one focused on the driver. Settle into the standard sport seats, everything feels tailored to the needs of the driver. The reach of the steering, seat height and amount of lean all conspire to create the perfect driving position. The ergonomic steering provides excellent grip for large or small hands. Enthusiast drivers would lament the loss of a physical handbrake in favor of an electronic one which takes some of the fun out of hot hatch hooning.

(SG-01, Paragraph 9)

Another notable observation was that steering was also used as an adjective, based on the following sample paragraphs:

Open the door and you are welcomed by Clark Plaid fabric with Alcantara bolstered seats for a nostalgic yet assuring feel when you drive it like you stole it. The red accents on the contrast stitching and ambient door trim accent lighting further remind you of the car's exciting nature. Sit on the driver's seat and hold the meaty flat bottom steering wheel and you'd find yourself wanting to awaken the monster inside. It comes with paddle shifters if you prefer to take shifting duties to your own.

(PH-01, Paragraph 4)

On B roads, the 'Sport' mode contributed significantly to the GTI's pure mechanical grip that seemed inexhaustible. Steering weight and accuracy was faultless and made the B roads so enjoyable we delayed our trip home just for another go at it.

(SG-01, Paragraph 22)

With multimedia connectivity fast becoming an important feature, the Vios finally moves to the digital age with full iPod connectivity through its integrated head unit. The six-speaker system is nothing to write home about, but it's good and crisp; and at least you can command it through your fingers via the steering wheel buttons. The cool-blue illumination on the stereo (also used on the multi-information display) does tend to wash out in bright sunlight, but it's not that of a big deal.

(PH-02, Paragraph 8)

The last two examples of terms found in all sample articles were the words suspension and hatch (see definitions on Table 1). The word suspension was found to function merely as a noun, unlike the terms drive and steering, as found in excerpts below: 
Stylistic Analysis of Philippine English and Singaporean English in Automotive Review Articles

Because of its compact-car roots, the Orlando has relatively small physical dimensions for an MPV. This makes it maneuverable and easy to park. And in most situations, the suspension can soak up typical Third World road imperfections.

(PH-03, Paragraph 11)

The suspension was set up to handle while not totally forgetting about comfort, though the 18-inch wheels with low profile tires and its rather high tire pressure recommendation do have their fair share of harshness. Cornering felt very confident as you pick up speed through the curves. The combined power and handling brought out a very agile car with a rigid Euro car feel to it. The brakes are very strong with a precise pedal feel.

(PH-01, Paragraph 9)

Expectedly, the steering is light, which is ideal for negotiating the city traffic and parking, but feedback is lacking. Grip from the comfort-oriented Yokohama dB Decibel tyres is just adequate, but it makes up for it by working together with the suspension to equip the Vios with a relatively quiet and comfortable ride.

(SG-02, Paragraph 12)

The car comes with a drive mode selector allowing you to choose between Normal, Eco and Sport modes. The driving modes tweak engine maps, transmission shift points and throttle response, since it's a GTI, I kept it on "Sport" mode most of the time. Sport suspension comes standard as well as uprated brakes with red colored calipers to match the engine and complement the tires.

(PH-01, Paragraph 7)

Similar to the word drive, the following excerpts depict occurrences of the word hatchwhich is a contraction of the word hatchback- as a noun both in its singular and plural forms:

Well, the recipe is simple and has endured through the years with each generation. The hatch sits four adults and is equipped with a decent boot. Shoehorn a powerful turbocharged engine under the bonnet and you can have quite a chuckle when driving point to point going about your tasks.

(SG-01, Paragraph 4)

The Volkswagen Golf GTI is considered as one of the original "hot hatches", tracing its existence dating back four decades. While there may have been other similar models that have preceded it, the Golf GTI is perhaps the only one that has stood the test of time for seven generations.

(PH-01, Paragraph 1)

One of the reasons behind the similarity in terms of automotive-related register is that automotive terms, especially neologisms, are universal. Automotive articles across the world, especially those in English, use the same terms in order to let readers understand that featured vehicles are generally accessible to them, regardless of their location. The second reason behind the results is that automotive terms are dictated not by a single society but by manufacturers themselves, even terms dating back to the $20^{\text {th }}$ century. For instance, the word hatch (formally known as hatchback and formerly known as lift back) refers to a vehicle whose rear door, 
including the glass, opens all the way, thus enabling drivers and passengers to utilize cargo space in the rear. Another notable term, suspension, simply refers to the mechanism that supports a vehicle and enables it to maintain its height regardless of driving conditions, such as rough roads, steep inclines, and plains. Lastly, the similarity in terms of register could be attributed to the desire of automotive writers to make their articles accessible not only to readers whose nationalities are similar to theirs but also to readers of other nationalities, since automotive articles are required to be written in English. The use of the said language makes automotive articles more coherent and cohesive, which are required in order to enable different readers (e.g. prospective vehicle buyers, automotive manufacturers, sales agents, and other similar readers) to understand not only the aesthetics of certain vehicle models but also technical specifications, which require a reader to decode not only the meaning but also the context of every automotiverelated technical term. Since automotive articles are technical and at the same time written to appeal to a general audience, automotive registers are required to be used by automotive writers, especially terms that originate from the latest automotive technologies introduced by various manufacturers (e.g. 4Matic by Mercedes-Benz, hybrid vehicles, EV [which stands for electric vehicles], dual clutch, and other similar terms).

\section{Grammatical Areas \\ Adjective Usage}

Another area that the current study explored was the use of adjectives in all sample automotive reviews. The adjective that the researchers focused on was those pertaining to vehicles, especially those referring to measurements and the number of cylinders in an engine or speeds in a transmission. Based on the adjectives found across all articles, the researchers created the following general categories of adjectives that apply to the automotive industry: a) Pertaining to quantity, b) Pertaining to quality, and c) Pertaining to origin.

Adjectives pertaining to quantity include specific measurements (such as how large an engine is or how long the wheelbase of a vehicle is) and quantities in numbers. Adjectives pertaining to quality refer to color, size, shape, road feel (a driver's perception of the way a vehicle responds to his/her driving style), and proper adjectives that describe the type of object the noun is (e.g. Volkswagen hatchback, Becker stereo, and Giorgetto Giugiaro and Paul Bracq designs; all examples are provided by the researchers). Finally, adjectives pertaining to origin refer to the place of origin of an object being modified. Examples of adjectives pertaining to quantity are found in the sample paragraphs below:

With the very same platform, very same 1.5-liter, 4-cylinder engine, and very same 4-speed automatic, you'll walk away thinking the all-new model to behave the exact same way as before.

(PH-02, Paragraph 10)

Providing the oomph for the new Vios is the familiar 1.5-litre 1NZ-FE four-cylinder engine that has been in service since 2002. Like before, it develops 107bhp at 6,000 rpm and 141Nm of torque at 4,200rpm.

(SG-02, Paragraph 10)

The highlighted adjectives above were found to be related to engine capacity, number of speeds in a transmission, number of cylinders in an engine, and to measurement of speed and power. A possible reason for the use of such adjectives is because of specificity and accuracy. 
Stylistic Analysis of Philippine English and Singaporean English in Automotive Review Articles

Since the automotive industry stresses the vitality of ensuring a long-lasting performance of their engines, it is necessary that accuracy of measurements should be observed.

Samples of adjectives pertaining to quality are found below:

The suspension was set up to handle while not totally forgetting about comfort, though the 18-inch wheels with low profile tires and its rather high tire pressure recommendation do have their fair share of harshness. Cornering felt very confident as you pick up speed through the curves. The combined power and handling brought out a very agile car with a rigid Euro car feel to it. The brakes are very strong with a precise pedal feel.

(PH-01, Paragraph 09)

With a wheelbase of 2,631mm, the GTI is considerably more spacious than some of its rivals in this category. Fitting four full sized adults in this hatch is easy peasy with plenty of knee room in the back. Boot capacity is a respectable 380 litres and will fit a decent amount of shopping with ease. Folding the seats further increases the capacity to 1,270 litres.

(SG-01, Paragraphs 15-16)

Apart from register and adjective usage, another common area in both SE and PE automotive reviews is pronoun usage.

\section{Pronoun Usage}

It was revealed through document analysis that all automotive review articles used a combination of first-person, second-person, and third-person pronouns. Focusing on the firstperson pronouns, I, we, my, and our were spotted across all articles. Both types of pronouns were found mostly in the introduction section of most articles, though a number of first-person pronouns were found in the middle and/or towards the concluding paragraph of almost every article. A possible reason behind the use of first-person pronouns could be the authors' desire to integrate their own experiences in driving the vehicles that they were assigned to evaluate in terms of performance, accessories, aesthetics, and other factors. Another reason behind the use of first-person pronouns by the authors could be attributed to the authors' desire to interact with their readers by narrating their experiences in driving the vehicles assigned to them. Hence, it offers a more convincing tone.

The following examples of occurrences of the first-person pronouns I, we, my, and our (including contractions of phrases such as I have and we have) depict the predominance of these types of pronouns over the second-person pronoun you:

For this test, the condition of the tires was well beyond encouraging, we didn't push the car to its limits. This is not to say that we didn't enjoy driving it. It was still a fun drive albeit an experience that was a little limited.

(PH-01, Paragraphs 11)

iPod / iPhone integration is among the best we've observed. The menu and search interface has negated the need for a CD player completely. The system allows you to scroll through your music library intuitively and even displays album art!

(SG-01, Paragraph 12) 
I have an embarrassing story to tell. Up till my first foray into the motoring journalism community, the Golf GTI was just another car. Sure, I've heard others rave about it for years since the Mk V version was launched but having not driven one personally up till then, I hadn't known what I was missing anyway.

(SG-01, Paragraph 1)

Fifty percent of the Philippine passenger car market-by any standard, a car that's as sellable as this is also the most influential. So, it shouldn't come as a surprise then that the allnew Toyota Vios is the most important car of the year. Forget cup car racing or DJ parties, I finally got behind the wheel of this hotly-anticipated vehicle and here's my verdict: damn, they got it right this time.

(PH-02, Paragraph 1)

Since then, this assembled-in-Thailand car has already gone through two generations. Two months ago, the local Toyota dealer Borneo Motors launched the all-new Vios. We take the new car out for a road test.

(SG-02, Paragraph 2)

The Cruze, which shares its platform with the Orlando, has a diesel engine. And so do the euro variants (with six-speed manuals!). Our advice? Put a diesel mill in the Orlando.

(PH-03, Paragraph 10)

Second-person pronouns were also found to be manifested in almost all articles, though not as prevalent as third- or first-person pronouns. These types of pronouns were more prevalent in the PE automotive reviews than in the SE versions. One possible reason behind the use of these types of pronouns may be the writers' intention to connect to the reader by writing in such a manner that resembles face-to-face interaction, which was more evident in the PE automotive reviews than in the SE counterparts. A second reason is probably the intention of the writers to instruct their readers on their expectations of the vehicle while retaining their technical writing style.

The following excerpts depict the use of second-person pronouns (including the use of possessive and reflexive forms of you, as well as contracted forms of you will):

Open the door and you are welcomed by Clark Plaid fabric with Alcantara bolstered seats for a nostalgic yet assuring feel when you drive it like you stole it. The red accents on the contrast stitching and ambient door trimaccent lighting further remind you of the car's exciting nature. Sit on the driver's seat and hold the meaty flat bottom steering wheel and you'd find yourself wanting to awaken the monster inside. It comes with paddle shifters if you prefer to take shifting duties to your own.

(PH-01, Paragraph 4)

Well, the recipe is simple and has endured through the years with each generation. The hatch sits four adults and is equipped with a decent boot. Shoehorn a powerful turbocharged engine under the bonnet and you can have quite a chuckle when driving point to point going about your tasks. 
Stylistic Analysis of Philippine English and Singaporean English in Automotive Review Articles

(SG-01, Paragraph 4)

With the very same platform, very same 1.5-liter 4-cylinder engine, and very same 4-speed automatic, you'll walk away thinking the all-new model to behave the exact same way as before. Well, I'm happy to tell you that it doesn't.

(PH-02, Paragraph 10)

It's a good thing the Orlando manages to look a little macho with its large grille, clean lines and chunky styling. It's a credit to the designers that no angle looks awkward, unless you count the bland side view. But that doesn't diminish the overall visual impact.

(PH-03, Paragraph 5)

The third-person pronouns found across all articles were used mostly to refer to the vehicles being reviewed, as depicted by the following excerpts; occurrences of it and its possessive form are emphasized below:

For this test, the condition of the tires was well beyond encouraging, we didn't push the car to its limits. This is not to say that we didn't enjoy driving it. It was still a fun drive albeit an experience that was a little limited.

(PH-01, Paragraph 11)

The new car inherits the same excellent interior as the current generation Golf. It is tastefully designed with faux carbon inserts around the centre console and around the cabin. New in the GTI is the mood lighting that emanates from the doors and foot wells that create a pleasant cosy environment for the driver and passengers at night.

(SG-01, Paragraph 10)

In terms of luggage space, Toyota claims the Vios can swallow up to four golf bags; and I won't dispute this since the Vios does appear to have one of the biggest and deepest trunks in its class. However, what I didn't like is the lack of storage compartments for passengers. Yes, there's a center console bin just in front of the shifter, but it's barely enough to fit an iPod nano. The center arm console, though tall, is quite narrow. And though there are the cup holders located in front of the driver and passenger air conditioning vents, it can't fit even a short-sized Starbucks latte. And even if you did manage to squeeze one in there, good luck in pulling it out without spilling a drop.

(PH-02, Paragraph 6)

The Toyota Vios first made its debut back in 2002. Specially developed for China, Taiwan and South-east Asia, it took over the baton from the rather bland Soluna - a budget entry-level subcompact car positioned below the Corolla family saloon.

(SG-02, Paragraph 1)

Because of its compact-car roots, the Orlando has relatively small physical dimensions for an MPV. This makes it maneuverable and easy to park. And in most situations, the suspension can soak up typical Third World road imperfections.

(PH-03, Paragraph 11) 
So yes, the Orlando is a worthy addition to the Chevrolet family, and as an MPV, it is more relevant to Asian families, a worthy alternative to the Toyota Wish and Honda Stream, and the its considerably more expensive rivals from Europe. It has an easy-going, yet masculine and selfconfident style that is refreshingly different from its rivals who seem be pretending to be either sporty (Honda Stream, Volkswagen Touran), or futuristic spaceships (Citroen Grand C4 Picasso), or purely functional and colourless (Toyota Wish).

(SG-03, Paragraph 14)

\section{Writer's Style}

Based on the gathered data, it was observed that the writing style of all authors were a combination of personal and technical. One of the reasons behind this observation was the presence of a combination of first-, second-, and third-person pronouns across all articles. A second reason was the inclusion of the authors' personal experiences in test driving the vehicles loaned to them for review. However, in terms of overall point of view, it was observed that the dominant point of view across all articles was the third-person point of view because the nature of the articles is technical more than personal due to the presence of automotive terminologies in the articles. Another style observed across all articles was the inclusion of historical and other background information on the featured vehicle models, which could possibly be due to the writers' desire to provide readers with more information on previous iterations of the models and at the same time help them compare the latest models with those from previous generations.

\subsection{Stylistic Differences of Philippine English and Singaporean English Article Contents}

The document analysis of all three (3) pairs of automotive reviews revealed that, in the SE versions of the automotive reviews included an abridged version of their positive and negative evaluations of the vehicles in review, in contrast to the PE versions. It was also observed that the SE automotive reviews tended to include direct competitors of the vehicles in review unlike the $\mathrm{PE}$ versions due to the presence of more vehicle marques and models available in the country of origin.

Apart from article contents, differences in terms of English variety conventions were also found in the SE and PE automotive reviews.

\section{English Variety Conventions}

Table 2. Lexical differences in terms of English variety conventions, as found across all articles.

\begin{tabular}{ll}
\hline SE & PE \\
1. Bonnet & Hood \\
\hline 2. Litre & liter \\
\hline 3. Colour & color \\
\hline 4. Metre & meter \\
\hline 5. Boot & trunk \\
\hline 6. Tires & Tyres \\
\hline
\end{tabular}


Stylistic Analysis of Philippine English and Singaporean English in Automotive Review Articles

Based on the table above, it was revealed that only five (5) words differed in terms of the English conventions. The majority of these words were different only in terms of spelling, with the remaining words being different in terms of variety of English, hood (bonnet in SE) and trunk (boot in SE) being the two terms. One of the reasons behind the differences is that the basis of PE is American English (AmE) while that of SE is British English (BrE). Another reason behind the sparseness in lexical differences is that many terms, especially automotive terms related to parts and other components (e.g. chassis, door, steering wheel, and other similar terms are provided by the researcher), are universal.

\section{CONCLUSION}

The current study analyzed the different stylistic similarities and differences between SE and PE automotive reviews. One major observation was that there were more stylistic similarities than differences. Focusing on writing style, it was observed in the majority of the articles that the authors included their personal experiences with the vehicles and that automotive registers were incorporated into all articles since most of these terms are familiar to readers, especially drivers, auto enthusiasts, and professionals in the automotive industry. As for the point of view, the most dominant was the third-person point of view because the subjects of the articles were on the vehicles.

This study could serve as a springboard for curriculum designers in automotive engineering and other related majors to create up-to-date programs that involve both the teaching of technology and the teaching of language skills, especially reading skills. Another pedagogical implication is the teaching of varieties of English and their grammatical structures- especially in the Asian context- because different varieties of English are utilized even in articles involving highly technical fields such as business, economics, and technology. It is also essential that different writing styles adopted by technical writers- especially in the automotive industry- be studied further by students and practitioners alike since the automotive industry evolves constantly in terms of innovations. Lastly, it is an imperative that the teaching of critical writing skills should be strengthened because automotive reviews require a writer to utilize facts and figures about the vehicle to evaluate its aspects.

A recommendation for further research is that more pairs of automotive reviews in SE and PE that tackle the same vehicle model and iteration be used for more thorough analysis and comparison of stylistic elements. Another recommendation for further research is the use of automotive reviews of the same model written in other Asian varieties of English, such as Malaysian English and Hong Kong English, and these must be written by Asians. It is highly recommended that other stylistic elements be explored in similar further studies.

\section{References}

Barbour-Lacey (2015, March 11). ASEAN case study: the auto industry and GM. ASEAN Briefing. Retrieved from http://www.aseanbriefing.com/news/2015/03/11/asean-case-study-autoindustry-gm.html

Crystal, D. (2003). English as a global language (2nd edition). Cambridge, UK: Cambridge University Press. 
Deocampo, M. F. (2012). An historical view of languages and the media in Singapore and in the Philippines: parallelism and cultural dimensions. Asian Journal of Literature, Culture, and Society, 6(2), 20-53.

Hyland, K. (2005). Metadiscourse. London, UK: Continuum.

Oxford Business Group (2015, September 6). The Philippines' auto industry riding high. BusinessWorld Online. Retrieved from http://www.bworldonline.com/content.php?section=Economy\&title=The Philippines\%E2\%80\%99-auto-industry-riding-high\&id=77780

Pakir, A. (2009). English as a lingua franca: analyzing research frameworks in International English, world Englishes, and ELF. World Englishes, 28(2), 224-235.

Rajagopalan, K. (April 2004). The concept of 'World English' and its implication for ELT. ELT Journal, 58(2), 111-117.

Remo, A.R. (2015, March 13). PH auto industry hits new record. Philippine Daily Inquirer. Retrieved from http://business.inquirer.net/188401/ph-auto-industry-hits-new-record

Salazar, D. (2014). Towards improved coverage of Southeast Asian Englishes in the Oxford English Dictionary. Lexicography ASIALEX, 1, 95-108.

Singapore Economic Development Board (2015). Emerging businesses. Retrieved from https://www.edb.gov.sg/content/edb/en/industries/emerging-businesses/emergingbusinesses.html

Tan, Y.Y. (2014). English as a 'mother tongue' in Singapore. World Englishes, 33(3), 319-339. 